\title{
The Cockayne Syndrome Natural History (CoSyNH) study: clinical findings in 102 individuals and recommendations for care
}

\author{
Brian T. Wilson, ${ }^{1,2}$, Zornitza Stark ${ }^{3}$, Ruth E. Sutton', Sumita Danda ${ }^{4}$, \\ Alka V. Ekbote ${ }^{4}$, Solaf M. Elsayed ${ }^{5,6}$, Louise Gibson7, Judith A. Goodship ${ }^{1,2}$, \\ Andrew P. Jackson ${ }^{8}$, Wee Teik Keng ${ }^{9}$, Mary D. King ${ }^{10,11}$, Emma McCann ${ }^{12}$, \\ Toshino Motojima ${ }^{13}$, Jennifer E. Murray ${ }^{8}$, Taku Omata ${ }^{13}$, Daniela Pilz ${ }^{14}$, Kate Pope ${ }^{3}$, \\ Katsuo Sugita ${ }^{15}$, Susan M. White ${ }^{3,16}$ and Ian J. Wilson ${ }^{2}$
}

\begin{abstract}
Purpose: Cockayne syndrome (CS) is a rare, autosomal-recessive disorder characterized by microcephaly, impaired postnatal growth, and premature pathological aging. It has historically been considered a DNA repair disorder; fibroblasts from classic patients often exhibit impaired transcription-coupled nucleotide excision repair. Previous studies have largely been restricted to case reports and small series, and no guidelines for care have been established.
\end{abstract}

Methods: One hundred two study participants were identified through a network of collaborating clinicians and the Amy and Friends CS support groups. Families with a diagnosis of CS could also self-recruit. Comprehensive clinical information for analysis was obtained directly from families and their clinicians.
Results and Conclusion: We present the most complete evaluation of Cockayne syndrome to date, including detailed information on the prevalence and onset of clinical features, achievement of neurodevelopmental milestones, and patient management. We confirm that the most valuable prognostic factor in CS is the presence of early cataracts. Using this evidence, we have created simple guidelines for the care of individuals with CS. We aim to assist clinicians in the recognition, diagnosis, and management of this condition and to enable families to understand what problems they may encounter as CS progresses.

Genet Med advance online publication 23 July 2015

Key Words: cerebro-oculofacioskeletal syndrome; Cockayne syndrome; CSA (ERCC8); CSB (ERCC6)

\section{INTRODUCTION}

Cockayne syndrome (CS) is a rare, autosomal-recessive disorder that was first described in 1936 by Edward Cockayne. ${ }^{1}$ Early descriptions of CS identified the cardinal clinical features of the disorder: microcephaly and growth failure. Other recognized features include hearing loss, cataracts, retinal dystrophy, and developmental delay. Dermal photosensitivity is often considered a key feature of the diagnosis, particularly after defects in transcription-coupled nucleotide excision repair (tc-NER) were identified in classically affected patients. ${ }^{2,3}$ However, the importance of this feature has long been questioned. ${ }^{4,5}$ Since then, and despite the discovery of the principal genes for CS (CSA/ERCC8, CSB/ERCC6), sensitivity of patient fibroblasts to ultraviolet (UV) C irradiation has been considered the diagnostic test of choice. ${ }^{6}$ Many case reports and series have been published over the years, including the landmark paper by Nance and Berry, ${ }^{7}$ which reviewed 140 cases from the literature. Although comprehensive, those authors noted that such review data may be subject to bias due to differences in reporting. Primary data have never been systematically collected from a large cohort of affected individuals, and no guidelines for care exist. This study aimed to provide a comprehensive clinical description of CS and recommendations for care, to assist clinicians in diagnosis and management, and when counseling families.

\section{METHODS}

Ethical approval was granted by the National Research Ethics Service Committee North East-Newcastle and North Tyneside 2. Individuals affected by CS were identified by an international

\footnotetext{
${ }^{1}$ Northern Genetics Service, Newcastle Upon Tyne NHS Foundation Trust, International Centre for Life, Newcastle upon Tyne, UK; ${ }^{2}$ Institute of Genetic Medicine, Newcastle University, International Centre for Life, Newcastle upon Tyne, UK; ${ }^{3}$ Murdoch Childrens Research Institute, Parkville, Victoria, Australia; ${ }^{4} \mathrm{Clinical}$ Genetics Unit, Christian Medical College, Vellore, India; ${ }^{5}$ Medical Genetics Center, Korba, Cairo, Egypt; ${ }^{6}$ Children's Hospital, Ain Shams University, Cairo, Egypt; ${ }^{7}$ Paediatrics \& Child Health, University College Cork, Cork, Republic of Ireland; ${ }^{8} \mathrm{MRC}$ Human Genetics Unit, Institute of Genetics and Molecular Medicine, University of Edinburgh, Edinburgh, UK; ${ }^{9}$ Clinical Genetics, Hospital Kuala Lumpur, Kuala Lumpur, Malaysia; ${ }^{10}$ Paediatric Neurology, Temple Street Children's University Hospital, Dublin, Republic of Ireland; ${ }^{11}$ School of Medicine and Medical Science, University College Dublin, Dublin, Republic of Ireland; ${ }^{12}$ Department of Clinical Genetics, Glan Clwyd Hospital, Rhyl, Denbighshire, UK; ${ }^{13}$ Division of Child Neurology, Chiba Children's Hospital, Chiba, Japan; ${ }^{14}$ Institute of Medical Genetics, University Hospital of Wales, Cardiff, UK; ${ }^{15}$ Division of Child Health, Faculty of Education, Chiba University, Chiba, Japan; ${ }^{16}$ Department of Paediatrics, University of Melbourne, Parkville, Victoria, Australia. Correspondence: Brian T. Wilson (brian.wilson@ncl.ac.uk)
}

Submitted 4 May 2015; accepted 30 June 2015; advance online publication 23 July 2015. doi:10.1038/gim.2015.110 
network of collaborating clinicians and through the Amy and Friends CS support groups. In addition, families with a diagnosis of CS could self-recruit to the study. Informed consent was obtained for inclusion of all participants. Individuals were included in the analysis if they had a diagnosis of CS confirmed by molecular genetic testing or impaired recovery a

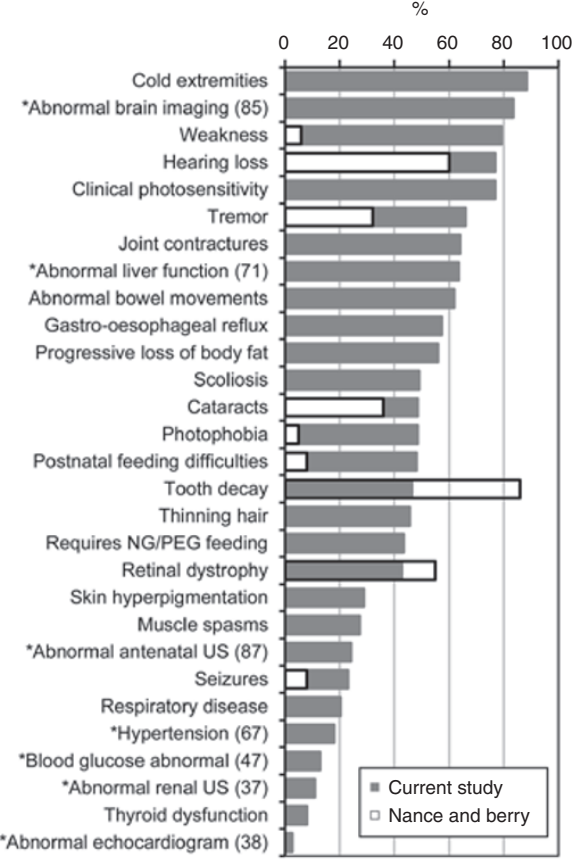

C
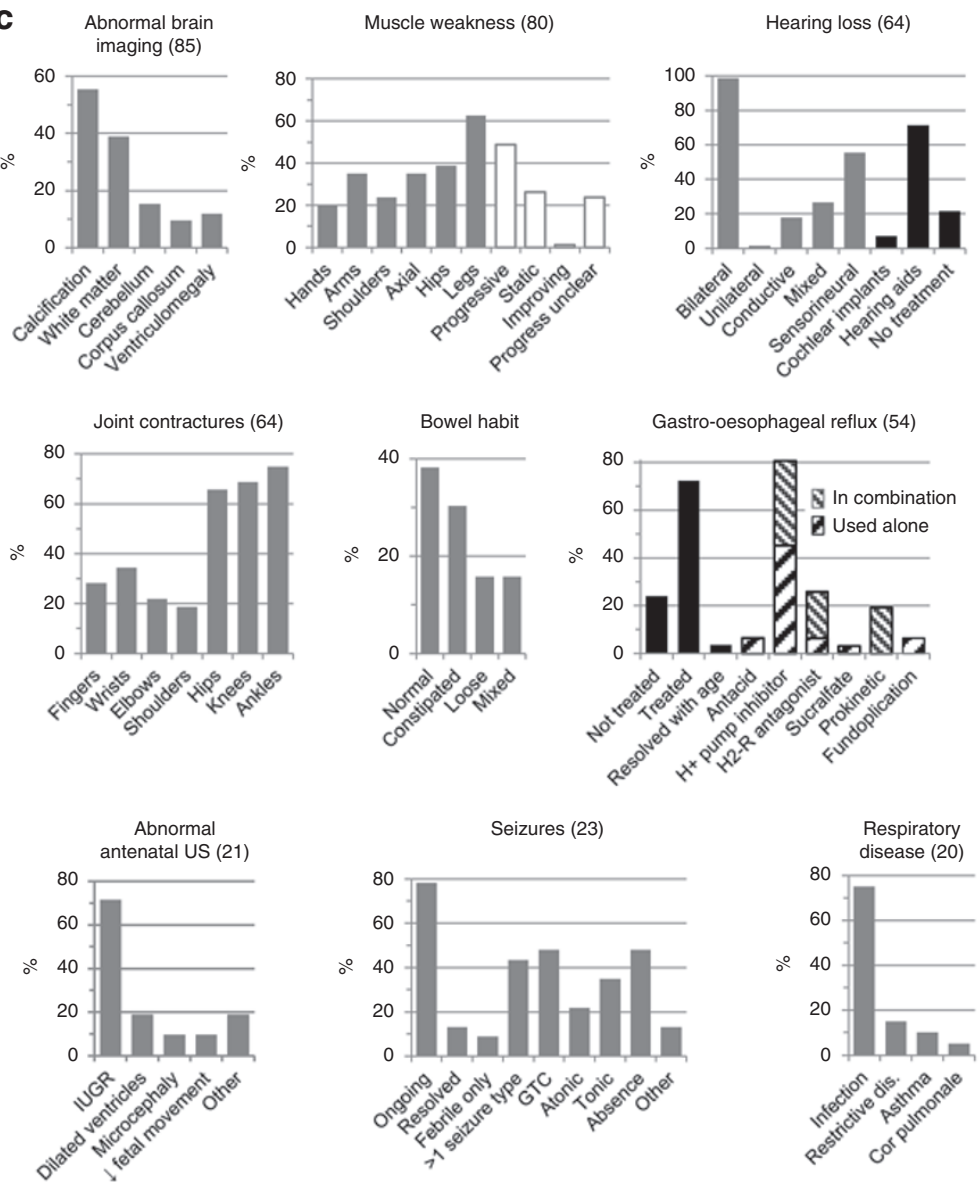

b

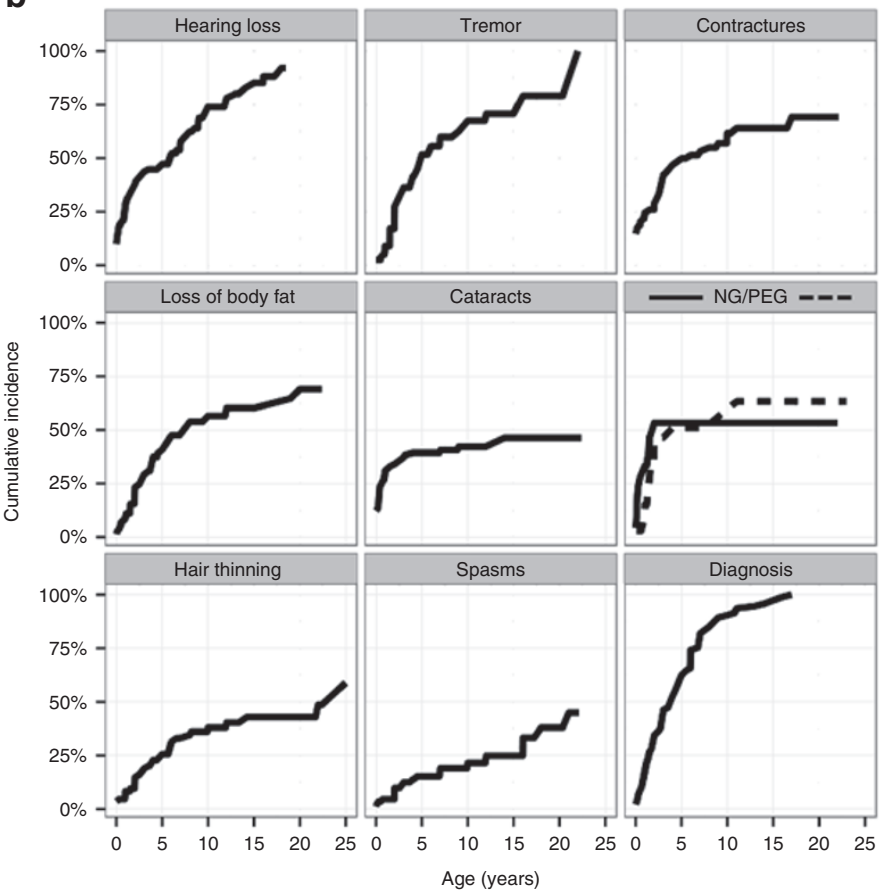

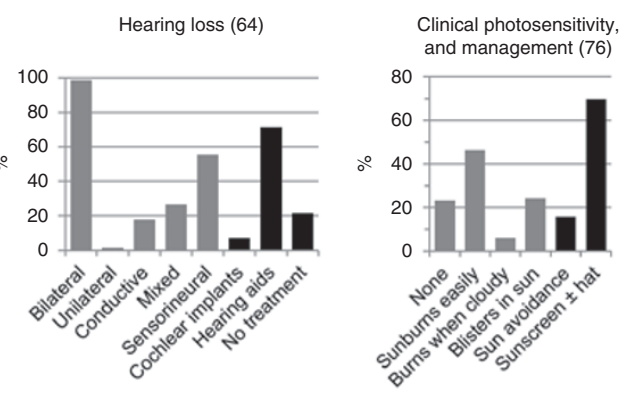

Tremor (66) and treatment (58)
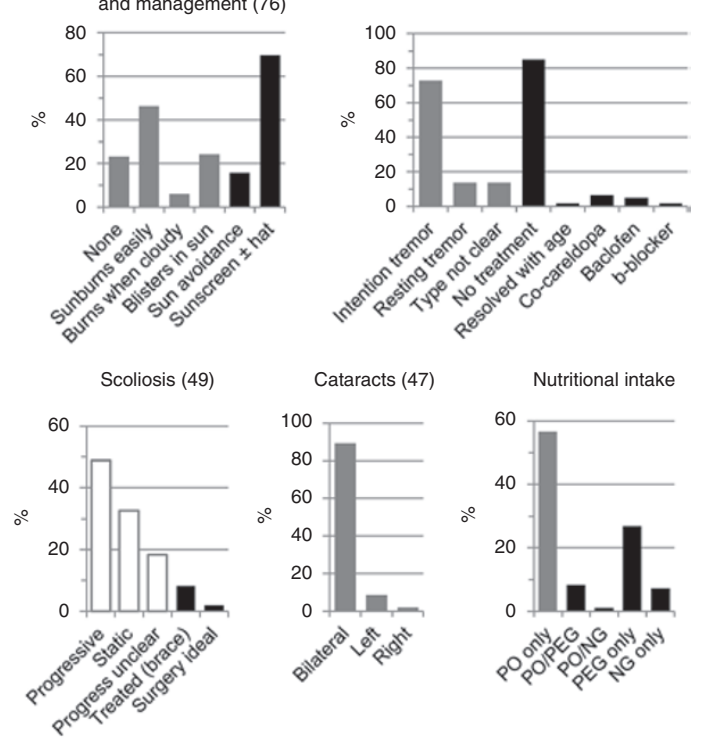

d

Diagnostic pathway (102)

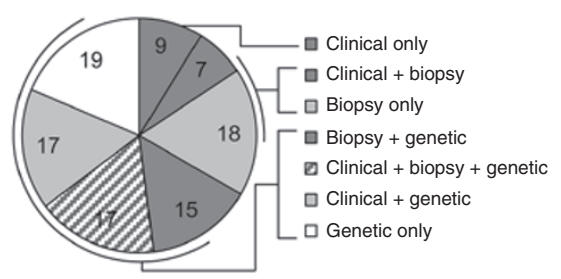


of RNA synthesis in fibroblasts following UVC irradiation. A small number of patients also were included without such confirmation if they had a clear clinical diagnosis of CS. Separate written, informed consent was provided for the publication of images. Primary data on different aspects of CS were systematically collected from affected families and their clinicians, as detailed in the Results. Wherever possible, clinicians reviewed original case notes, laboratory reports, and specialist interpretations for investigations such as imaging. All survival curves are Kaplan-Meier estimates; $P$ values were obtained using Mantel-Haenszel tests against the hypothesis that there is no difference in survival between groups.

\section{RESULTS}

One hundred two patients from 81 families across 4 major ethnic groups were recruited to the study (Supplementary Figure S1A online), comprising 44 females and 58 males. The mean age of recruited individuals was 11.5 years (range: 3 months to 39 years; Supplementary Figure S1B online). All were microcephalic and had growth failure leading to a proportionate short stature. At the time of analysis, 28 individuals had died; the mean age at death was 8.4 years (range: 17 months to 30 years; Supplementary Figure S1B online). Data on the prevalence of clinical features within our cohort are summarized in Figure 1a, together with detailed information on cumulative incidence (Figure 1b), manifestation, progression, and management of these features (Figure 1c). Genetic confirmation of CS was available in 40 pedigrees: CSB mutations were found in 28 (70\%), CSA mutations in 11 (27.5\%), and XPD mutations in $1(2.5 \%)$. Numerators/denominators appear in the text where information does not relate to the entire cohort (e.g., when age at onset is required for the analysis). Recommendations for care based on our findings appear in Table 1.

\section{Antenatal and birth history}

Eighty-seven of the 102 patients in the cohort were evaluated antenatally using ultrasound. Abnormalities were detected on 21 scans: prenatal growth restriction was noted in 15 , with a female-to-male ratio of $2: 1$. While the birth weight of all males identified in this way fell between the 0.4 th and 2 nd centiles for gestational age, this was between the 2 nd and the 91 st centiles for all females. Enlarged cerebral ventricles were noted in four cases and microcephaly in two cases.

Information on mode of delivery was available for 90 births: 27\% were Caesarean delivery, $4 \%$ were assisted and 69\% normal vaginal deliveries. Birth weights were almost entirely within the normal range (0.4th-99.6th centiles) and, based on available birth head circumference data, most individuals with CS were normocephalic (Supplementary Figure S1C,D online). Our data demonstrate that the first occurrence of CS in any family is extremely difficult to diagnose antenatally or at delivery.

\section{Facial phenotype}

Early in life, children with CS may look completely normal (Figure 2). The facial features of some children may be crowded, or the eyes may appear small. What is considered the typical facial appearance, with a loss of subcutaneous and orbital fat making the nose more prominent and giving the eyes a sunken appearance, only develops with time. However, the facial phenotype may be much more subtle. Some individuals develop a sunken-eyed appearance without loss of subcutaneous fat. In addition, children with CS may hold their upper limbs in a flexed position.

\section{Cardinal features: growth failure and microcephaly}

Postnatal growth failure and microcephaly in CS are well documented. ${ }^{78}$ In our cohort, growth parameters at birth were largely within the low normal range (median weight $-0.76 \pm 1.20 \mathrm{SD}$; occipital frontal circumference $-1.17 \pm 1.29$ SD). Postnatally, individuals with CS develop microcephaly and small stature (median occipital frontal circumference -7.34 $\pm 2.96 \mathrm{SD}$; height $-6.62 \pm 3.09 \mathrm{SD}$; weight $-6.62 \pm 4.79 \mathrm{SD}$; Figure $3 \mathrm{a})$. It is worth noting how wide the variation, particularly in height and weight, can be. Weight may initially fall across the centiles but continue to skim the bottom of the normal range early in life, or may rarely be within the normal range (Supplementary Figure S1E online). In some cases weight decreases rapidly below the normal range (Supplementary Figure S1F online). Microcephaly in CS has been described as progressive. This is a misnomer, which is unhelpful in discussions with families. Head circumference, as with other parameters, may be within the normal range early in life, but growth velocity rapidly declines to growth arrest, often between 1 and 2 years of age (Supplementary Figure S1G online). Height and weight also are arrested, though this occurs slightly later.

\section{Hearing and vision}

Hearing and visual problems are common in CS. It has been suggested that sensorineural deafness should be considered a feature of the disorder. ${ }^{9}$ Where detailed assessment of hearing loss was available, however, we found $44 \%$ (20/45) had conductive or mixed hearing loss. Whichever type of hearing loss is present, it is almost exclusively bilateral. This presents in $21 \%$ $(14 / 68)$ of cases neonatally and in $84 \%$ by age 10 years. While most affected individuals wear hearing aids, several have successfully received cochlear implants. This may be especially

\footnotetext{
Figure 1 Clinical features in Cockayne syndrome (CS). (a) Clinical features in our cohort in order of decreasing frequency. The asterisk indicates that the denominator is dependent on an investigation being performed; for example, 71 patients had serum analysis of liver function. Our data are compared with those presented by Nance and Berry (the values shown are independent, not cumulative). The considerable differences reflect the fact that our data have been systematically collected from primary sources, whereas the review was largely based on published case reports. (b) Cumulative frequency of features, corrected for survival. (c) Detailed information on clinical features, ordered as in (a). Grey bars are descriptive, white bars illustrate clinical progression, and black bars indicate management. $n$, number of patients in each analysis. (d) Chart illustrating how participants reached their current diagnosis. For "biopsy only," "biopsy and genetic," and "genetic only," this indicates that a confident clinical diagnosis was not given beforehand.
} 
Table 1 Recommendations for care

Recommended active follow-up/surveillance

Diagnosis

Named clinical input (should be easily accessible)

Annual surveillance

列

Management for:

Hypertension

GERD

Tremor

Recurrent pneumonia

Low temperature/unexplained symptoms

If unclear whether weight loss is due to inadequate intake or pathological subcutaneous fat loss

AVOID (may be fatal)

Exercise extra vigilance

Routine investigations that are commonplace but not indicated without clinical symptoms

CT/MRI of brain

Echocardiography

Renal ultrasound
First-line molecular testing of CSA and CSB

Molecular testing of ERCC5 if COFS phenotype and CSA/CSB are normal

Coordinating clinician (pediatrics, genetics if adult)

Dietician (before supplementary feeding required)

Physiotherapist/occupational therapist

Cataracts (annual/biannual until 4 years old)

Retinal evaluation (lifelong)

Hearing assessment

Review by named clinician, including:

Blood pressure, serum U\&Es if symptomatic

Liver function tests

Blood glucose (from 10 years old)

Review by physiotherapy

Consider:

Amlodipine \pm ACE inhibitor

Proton pump inhibitor \pm prokinetic agent

Co-careldopa

Referral for swallow assessment

Thyroid function tests

Titrate NG/PEG feeds against weight gain, avoiding rapid increases in volume

Metronidazole (and related antibiotics)

Opioids (consider starting at $1 / 3$ dose)

Sedatives

Typical findings are not pathognomic, prognostic, or clinically actionable

Reported abnormal in only one asymptomatic individual (dilatation ascending aorta)

Renal calculi only actionable finding to date

ACE, angiotensin-converting enzyme; COFS, cerebro-oculofacioskeletal; $C T$, computed tomography; GERD, gastroesophageal reflux disease; MRI, magnetic resonance imaging; NG, nasogastric; PEG, percutaneous gastrostomy; U\&E, urea and electrolytes.

useful in individuals unable to wear both hearing aids and glasses at the same time.

Cataracts and photophobia are equally common, occurring in $48.5 \%$ of our cohort. In most cases cataracts are bilateral, and $86 \%$ (38/44) occur by age 4 years. The cause of photophobia in CS remains elusive, but parents should be aware that children with CS may require sunglasses or shade to minimize this. Retinal dystrophy has long been recognized as a feature, ${ }^{7}$ typically with saltand-pepper pigmentation; this is present in $43 \%$ of our cohort.

\section{Musculoskeletal}

Weakness and joint contractures are major features of CS. The lower limbs are more often affected than the upper limbs, particularly by contractures (in 64\%), which may make caring for a child with CS difficult (e.g., changing diapers). Although 26\% $(11 / 42)$ of contractures present neonatally, they may occur throughout life. Physiotherapy input is likely to be required, and orthoses (e.g., splints) may prove useful in managing contractures. Weakness has been infrequently reported previously ${ }^{7}$ but affects $79 \%$ of our cohort and is progressive in $49 \%$ (39/80) of those. Similarly, scoliosis affects $48 \%$ of study participants and is progressive in $49 \%(24 / 49)$ of those. Treatment with a brace was required in four cases; in one patient the severity of scoliosis was thought to warrant surgical intervention, but the associated risks outweighed this.

\section{Gastrointestinal and nutritional}

Feeding and nutritional status are significant concerns in CS. Forty-eight percent of our cohort experienced postnatal feeding difficulties, and 57\% developed gastroesophageal reflux disease. This is managed effectively in most cases using proton pump inhibitors, either alone or in combination with a prokinetic agent or H2-receptor antagonist. In some individuals fundoplication may be required. Often, before a diagnosis is made, the growth of 
a

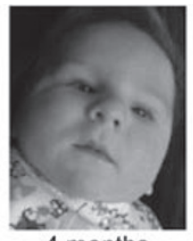

b

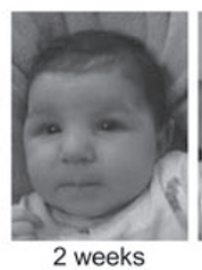

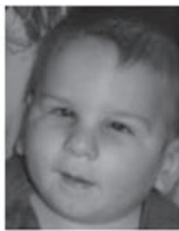

1 year

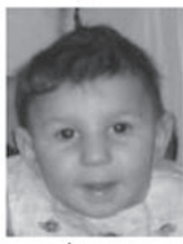

1 year

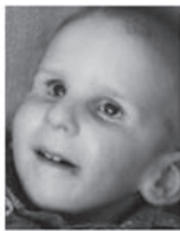

3 years

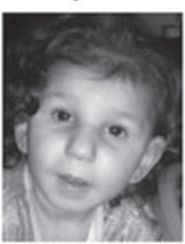

2.4 years

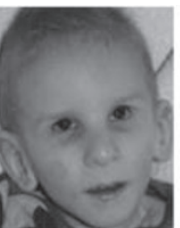

3.4 years

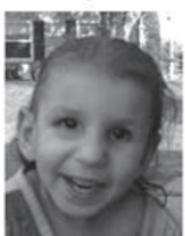

3.75 years

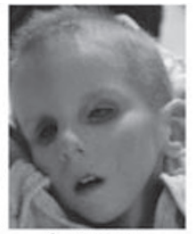

3.5 years

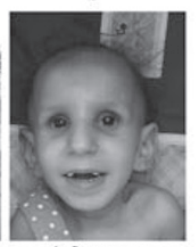

4.6 years

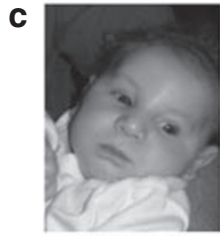

2 months

e

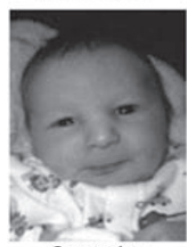

3 weeks

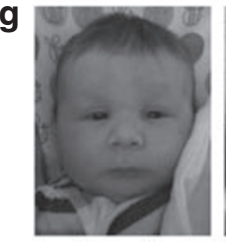

1 month

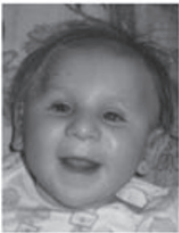

1 year

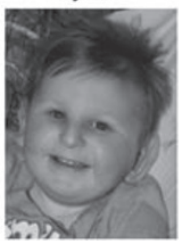

1 year

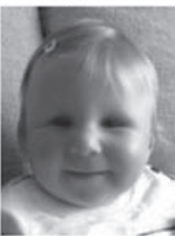

1 year

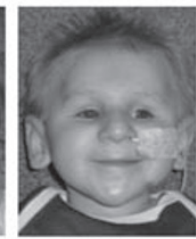

2 years

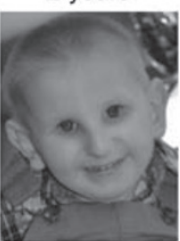

2 years

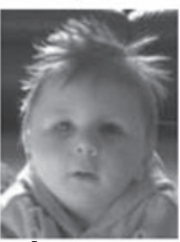

h: 1 year d

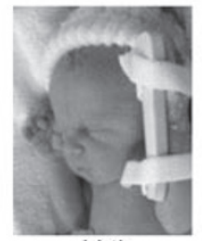

birth

$\mathbf{f}$

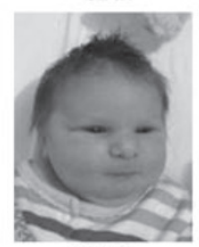

birth

i

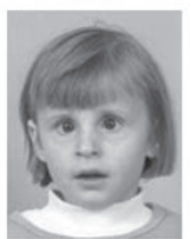

3 years

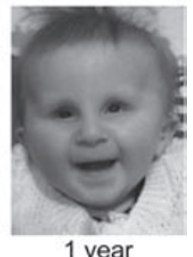

1 year

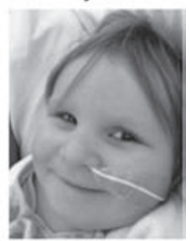

1.25 years

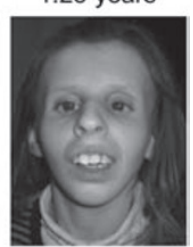

8 years

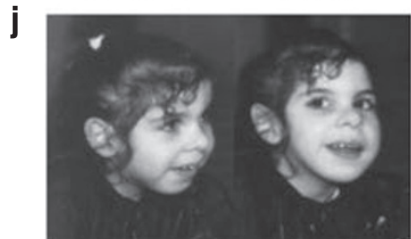

5 years

k

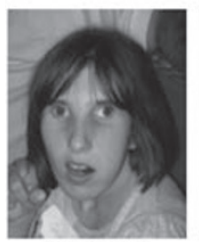

19 years

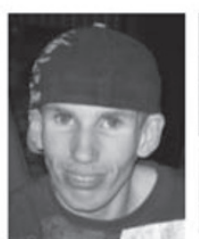

20 years

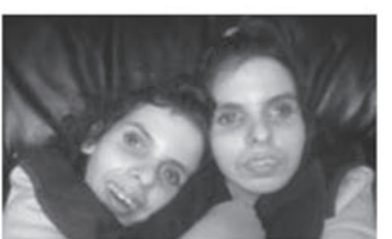

17 years

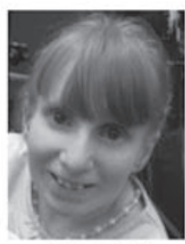

21 years

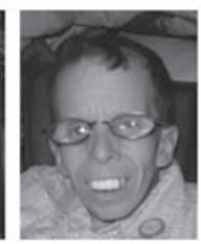

25 years

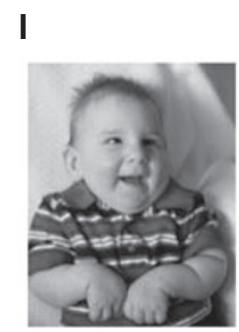

Figure 2 Changing features in Cockayne syndrome (CS). (a-I) Study participants (ages shown). Some individuals look completely normal early in life, whereas others may have subtle clues to the diagnosis, including crowded facial features and small eyes. While loss of subcutaneous fat, with development of the classic CS appearance $(\mathbf{a}, \mathbf{b}, \mathbf{j})$, is not a feature in all, a sunken appearance to the eyes often develops with a loss of orbital fat. Patients $\mathbf{g}$ and $\mathbf{h}$ are sister and brother. The girls appearing in $\mathbf{j}$ are identical twins. Older individuals with CS are shown in $\mathbf{k}$. Note also the flexed upper limb posture sometimes seen in patients with CS (I).

children with CS falls across the centiles. This may give rise to concerns that dietary intake is inadequate. The clinical status of the child with CS, rather than growth, should guide the clinician as to whether supplementary nasogastric or percutaneous gastrostomy feeding is required. Of patients who commenced nasogastric feeds, $63 \%$ (15/24) transitioned to percutaneous gastrostomy 


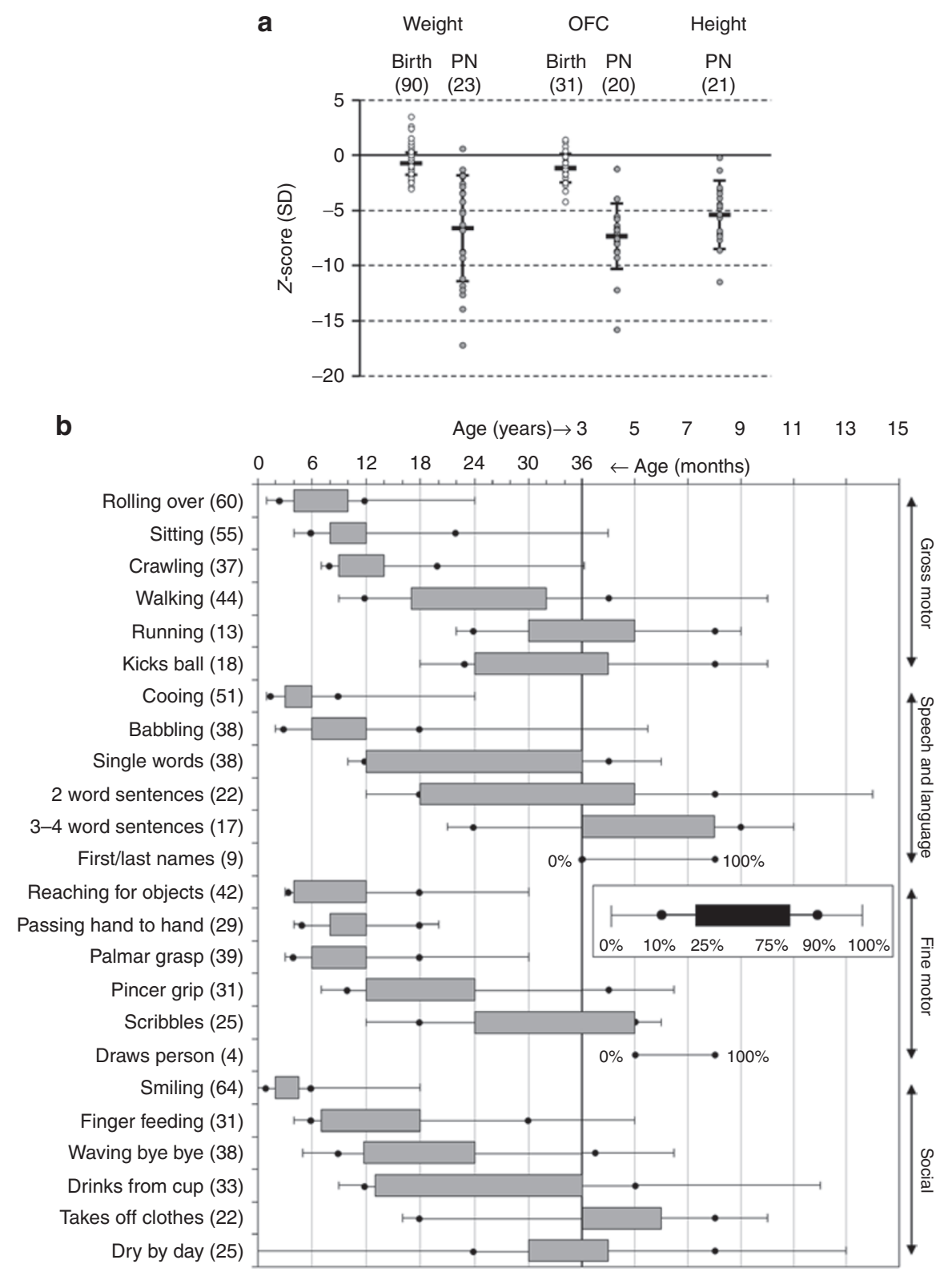

Figure 3 Growth and development in Cockayne syndrome (CS). (a) Growth parameters are in the low normal range at birth, but rapid reduction in growth velocity to growth arrest occurs postnatally (PN). Z-scores were calculated using the LMS method on 1990 British Growth Reference data. $n$, number of patients plotted. ${ }^{32}$ Mean values \pm 1 SD are shown. (b) Developmental ranges in CS. Ages at which participants achieve developmental milestones are shown. $n$, number of patients for whom age at attainment was available.

feeding, with a mean interval of 7 months. Further concerns arise in individuals who lose subcutaneous fat (56\%). Feed volumes may be increased to counter this. Subcutaneous fat loss is inexorable when present, however, and increased calorie intake does not seem to prevent this. In addition, at this stage in their condition, the stomachs of individuals with CS seem to be less able to accommodate increases in feed volume, which may cause vomiting. Again, clinicians should be guided by the clinical status of the patient. We suggest adopting a commonsense approach, trialing a modest increase in feeds while monitoring weight gain to establish whether existing intake is adequate. Subcutaneous fat loss may also lead to leakage of stomach contents around percutaneous gastrostomy tubes, reducing intake and causing skin irritation. Because patients may be receiving almost continuous feeds, this can be socially disabling as well as distressing for families. We advocate early management of such leakage because subcutaneous fat loss is unlikely to improve. Bowel disturbance is also common in CS and may manifest as constipation and/or loose stools. A number of children with loose stools have experienced particular problems with difficult-to-manage diaper rash. It is important to address this early to prevent deterioration of skin integrity. 


\section{Liver}

Hepatic dysfunction in CS has been previously noted, ${ }^{10}$ and in some cases it has been associated with cholestasis. Deranged liver function tests were found in $63 \%$ of patients in our cohort who had been tested $(n=71)$. Typically, transaminases are mildly elevated and may progressively increase. The underlying reason for this phenomenon is unclear. Annual serum liver function tests may be useful clinically, allowing differentiation between an increasing baseline and acute hepatic injury. We have reported elsewhere that metronidazole causes acute hepatic failure in CS, which may be fatal and should be avoided. ${ }^{11}$

\section{Cardiovascular, respiratory, immunology}

Cold extremities, representing poor peripheral circulation, are the most common non-cardinal feature of CS (88.4\%). This may be developmental in origin; teratomas generated using induced pluripotent stem cells derived from CS fibroblasts have very poor vasculature. ${ }^{12}$ In keeping with this, venous access is often difficult to achieve in individuals with CS (reported in 70\%), and repeated attempts are a frequent cause of distress for both patients and families. Venous access should ideally be undertaken in patients with CS only by experienced staff. Thirty-eight of our participants had an echocardiogram. This was abnormal in only one case, showing cardiac failure. There have been two previous reports of cardiac abnormalities in CS: dilated cardiomyopathy ${ }^{13}$ and dilation of the ascending aorta. ${ }^{14}$ Respiratory problems affected 20 patients; 15 developed respiratory infections, thought to be due to aspiration. Recurrent respiratory infections in individuals with CS should therefore trigger speech and language therapy referral for swallowing assessment. Immune problems are not a feature of CS, and affected individuals do not experience any increased incidence of infections compared with the general population; routine immunology input is therefore not required. Other respiratory problems in our cohort include restrictive lung disease and asthma.

\section{Renal and blood pressure}

To date, there have been few reports regarding renal manifestations in CS. Thirty-seven individuals in our cohort had renal ultrasound; this was abnormal in four, including unilateral hypoplastic (but functional) kidney, abnormal kidney shape, and renal calculi. A single patient developed nephrotic syndrome at 6 years. Sixty-seven participants had blood pressure evaluation; $18 \%(n=12)$ were hypertensive. We therefore recommend blood pressure evaluation annually and at each admission in CS. One patient was managed using a $\beta$-blocker alone. At least one patient was refractory to this therapy. Others were managed effectively using amlodipine, alone or in combination with an angiotensin-converting enzyme inhibitor or angiotensin-II receptor antagonist.

\section{Endocrine}

Forty-seven of 102 participants had laboratory assessment of blood glucose, with investigation type being dependent on local practice. Of those, $13 \%(n=6)$ had abnormal glucose metabolism. This affects older patients with CS and can progress from impaired glucose tolerance to required administration of insulin. The earliest occurrence of diabetes requiring treatment occurred at age 16 years. Autoimmune investigations, when performed, were normal. Although it is unclear how many participants were tested, eight had hypothyroidism; whether this was present from birth is unknown.

\section{Neurological}

Abnormal brain imaging is a well-recognized feature of CS. ${ }^{7,8,15}$ Computed tomography and/or magnetic resonance imaging had been performed for 85 individuals in our cohort; abnormalities were detected in $83.5 \%$ (71/85). Calcification was present in 55\% (47/85). This principally affects the basal ganglia, but diffuse cortical calcification may also be seen. This may represent more complex mineralization, rather than simple calcification, since microscopic examination also reveals the coincident presence of iron. ${ }^{16}$ White matter changes $(38 \%, 33 / 85)$ comprise dysmyelination or hypomyelination. Cerebellar hypoplasia or atrophy, enlarged cerebral ventricles, and thinning of the corpus callosum may also be seen. While calcification of the basal ganglia may point toward this diagnosis, the intracranial findings in CS are not pathognomonic, prognostic, or clinically actionable, and imaging may therefore be of limited value to the patient.

Seizure disorders and tremor have been infrequently reported previously, ${ }^{7}$ but they affect 23 and $66 \%$ of our cohort, respectively. In the majority of affected patients seizures are ongoing, and there is no predominant seizure type. Tremor among our patients is most often an intention tremor; in some cases onset is associated with apparent developmental regression, as children become less skilled in fine and gross motor domains. Despite a previous report showing a good response to carbidopa-levodopa in individuals with CS with tremor, ${ }^{17}$ very few participants received any treatment for this. Progressive neurodegeneration also has been described as a prominent feature in CS. We are not yet able to provide a detailed assessment of this in our cohort.

\section{Neurodevelopment}

Developmental delay is frequently reported in CS, although what families and clinicians might expect to observe is unknown. We therefore collected data on the age at which our cohort reached easily recognized developmental milestones (Figure 3b). Early development in particular may seem normal. Please note that these data represent only those individuals reaching each milestone, when the age at attainment was also known; some children with CS never develop the ability to roll over.

\section{Dermatological and dental}

In three individuals the presence of photosensitivity was unclear because of preemptive management. Twenty-three of 99 study participants have no dermal photosensitivity. Forty-six of 99 sunburn easily, but more explicit photosensitivity is present in only 40; these participants sunburn on cloudy days or 
blister following exposure to sunlight. Sun avoidance prevents issues among the latter group, whereas the former group effectively use simple measures, such as the use of sunscreen and hats. Individuals with CS are not at increased risk of developing skin cancers. Thinning hair and skin hyperpigmentation have been noted in previous reports and are present in 46 and 29\% of our cohort, respectively, but they have no clinical significance. There is no evidence to support routine dermatology input in CS. Review may be required upon the initial presentation of more marked photosensitivity, however, and may be useful in individuals with dry or itchy skin. Dental caries, which likely represents enamel hypoplasia, ${ }^{9}$ has been reported more frequently in the literature than they occur in our cohort (46.4\%). Nevertheless, families should be encouraged to maintain regular dental review so that problems relating to enamel hypoplasia can be addressed early.

\section{Medication}

We already highlighted (above and elsewhere) that metronidazole causes acute hepatic failure in CS, which may be fatal. The clinical presentation can be similar to an untreated paracetamol overdose, with extremely increased transaminase concentrations and significant clotting abnormalities. Metronidazole is therefore absolutely contraindicated in CS. Antibiotics of the same class should also be avoided, or used with extreme caution and close monitoring of liver function. There is no evidence of toxicity associated with drugs metabolized in the liver in general.

An exaggerated response to sedative and opioid medications-from respiratory depression to blunted affect lasting several days following administration of codeine-was reported in five cases.

\section{DISCUSSION}

CS is a rare condition that may be extremely difficult to recognize early in life. It has historically been considered a DNA repair disorder, yet the only feature that can be confidently attributed to defective tc-NER is photosensitivity. This problem has been highlighted by the identification of patients with UV-sensitive syndrome caused by mutations in CSA or CSB..$^{18,19}$ These patients have a DNA repair defect identical to that in those with CS, but they have normal growth and development and lack microcephaly or features of premature pathological aging. A defect in transcription, independent of tc-NER, has been suggested as the cause of CS-specific pathology ${ }^{20}$ but similarly suffers from the reports of patients with $C S A / C S B$ mutations with UV-sensitive syndrome and cannot explain why the particular features seen in CS occur together. Various additional roles for CSB have been tentatively suggested: in modulating the activity of base excision repair enzymes, ${ }^{21}$ in stabilizing mitochondrial DNA repair, ${ }^{22,23}$ in hindering the repair of covalent DNA-DNA topoisomerase I complexes, ${ }^{24}$ and in maintaining telomere stability. ${ }^{25} \mathrm{~A}$ role for the CSB-PGBD3 piggyBAC fusion protein has also been suggested; this protein is purported to regulate gene expression through interaction with chromosomal activator protein-1 proteins at tumor-promoting antigen response element motifs. ${ }^{26}$ However, there is no evidence that any of the additional processes in which CSB may be involved are significant in producing the CS phenotype, and no link between CSA and these processes has been identified. Any unifying pathogenic mechanism underlying the clinical features in CS is currently unknown, despite the detailed characterization of tc-NER.

\section{Incidence}

The incidence of CS in Western Europe is estimated at 2.7 per million live births. ${ }^{27}$ This may be an underestimate due to difficulties in reaching a diagnosis, in particular the reliance on dermal photosensitivity as a diagnostic feature. There are also examples of very high carrier frequency (1:15) in genetically isolated communities in Israel. ${ }^{28,29}$

\section{Diagnosis}

Modified criteria for the diagnosis of CS have recently been suggested (Supplementary Figure S2A online). Growth failure, microcephaly, and developmental delay were considered mandatory. However, our data suggest that developmental delay may be a poor discriminating factor for early diagnosis, since development may initially be within normal limits. We evaluated how effective the suggested minor criteria would have been in reaching a diagnosis of CS in our cohort. To do this, we used caries as a proxy for enamel hypoplasia and assumed that all patients had evidence of enophthalmos at diagnosis (since we had no objective evidence on this). Using these criteria, $36 \%$ of our cohort would have been diagnosed as having CS (Supplementary Figure S2B online), and only 20\% would be diagnosed if milder presentations of photosensitivity were not recognized. The ineffectiveness of these criteria reflect the variability in phenotype between patients with CS, which should be taken into account during clinical evaluation.

We suggest that CS should be suspected in any child with postnatal growth failure, microcephaly, and any two of the following: persistently cold hands and feet, bilateral hearing loss, dermal photosensitivity, (intention) tremor, joint contractures, progressive loss of body fat, cataracts, or typical facial features. Using these criteria increases clinical recognition of CS in our cohort to around $90 \%$.

Establishing an unequivocal diagnosis in CS is important (i) for proper management of the patient, (ii) to empower families in caring for affected individuals, and (iii) to provide accurate genetic counseling to parents and siblings. Historically, the ability of patient fibroblasts to resume RNA synthesis following UVC-induced DNA damage has been the mainstay of diagnosis in CS. However, this requires a skin biopsy, may produce equivocal results, and does not furnish the clinician with optimal information for genetic counseling. Furthermore, in our experience, if a clear tc-NER defect is present, a molecular genetic diagnosis can be achieved, suggesting that skin biopsy is usually unnecessary. DNA sequencing may also resolve the diagnosis in cases with equivocal tc-NER results. Therefore, we 
$\triangle$ Early cataracts $(<3 \mathrm{y})$

No early cataracts
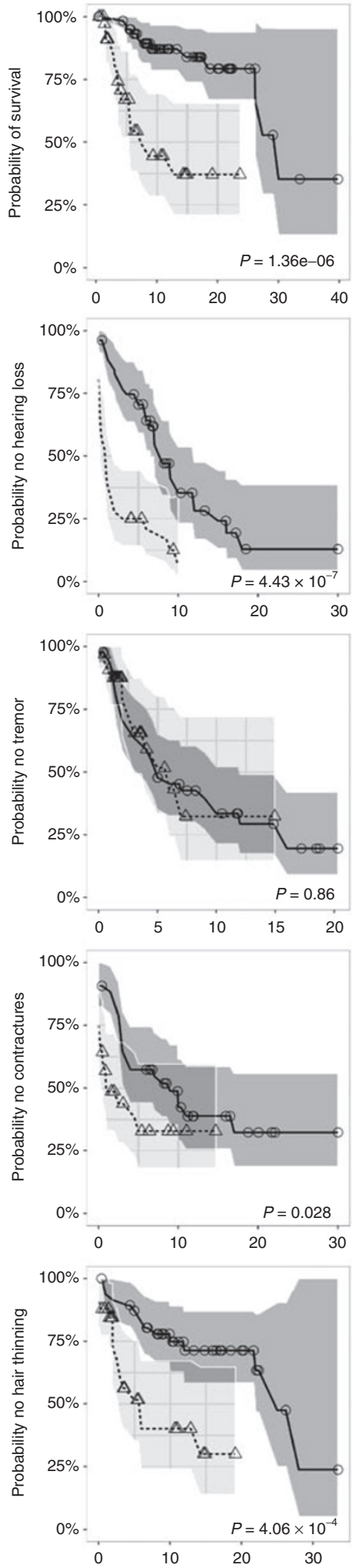

b
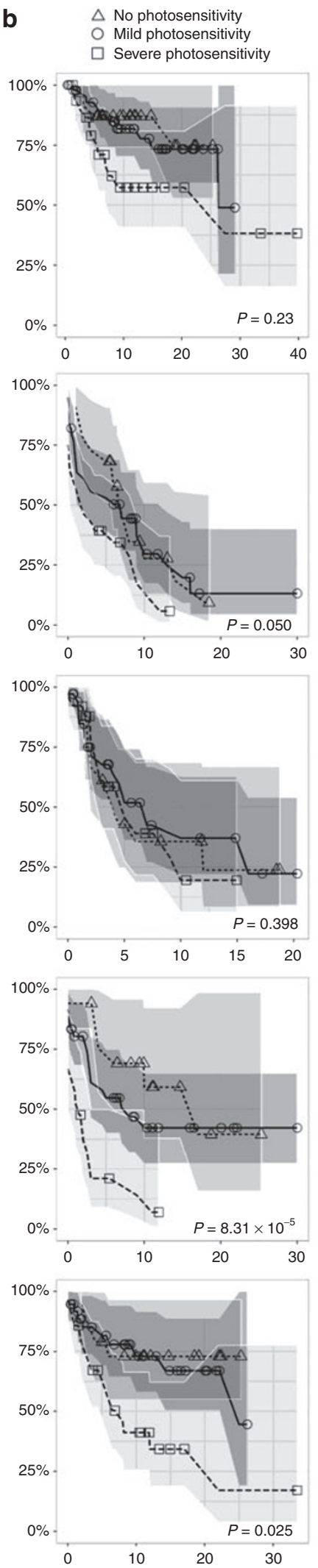

C $\triangle C S B / E R C C 6$ mutation

O CSA/ERCC8 mutation
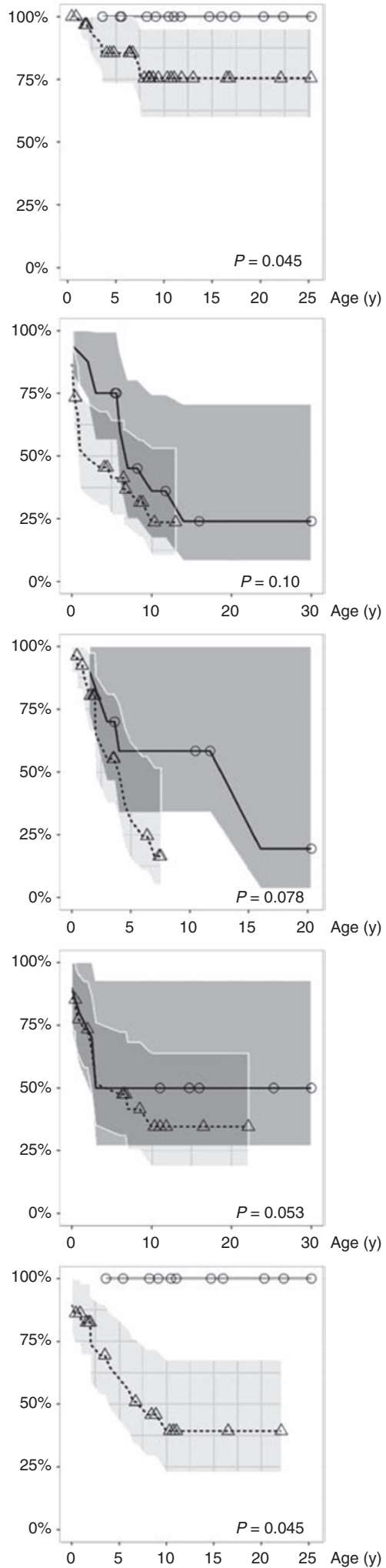

Figure 4 Prognostic factors in Cockayne syndrome (CS). The occurrence of cataracts before 3 years of age is the single most valuable prognostic factor in CS (a), having statistically extremely significant associations with reduced survival and shorter intervals to development of several other clinical features associated with reduced quality of life. Severe photosensitivity is associated with earlier onset of contractures, but not other factors affecting quality of life, or mortality (b). Having mutations in CSB (rather than CSA) has a borderline significant association with reduced survival only (c). For all plots, Kaplan-Meier estimates of the survival curve and $95 \%$ confidence intervals are shown. Statistical tests are Mantel-Haenszel tests of difference in the survival curve between groups. 
have adopted first-line molecular genetic testing of CSA and CSB in suspected cases of CS, which avoids the uncertainty of equivocal biopsy results, provides clear recurrence information for parents, and allows definitive prenatal testing and testing for potential carriers and their partners, if this is desired. We have successfully diagnosed patients using DNA extracted from blood, mouthwash, and dried bloodspot samples, avoiding the need for skin biopsy.

\section{Classification and prognosis}

Since Nance and Berry ${ }^{7}$ published their extensive review of the CS literature, clinicians have attempted to partition affected individuals into clinical subtypes. This is often confusing for families. In reality there is a clinical spectrum for each feature of CS, and affected individuals occupy a unique position on each. Phenotypic discordance between siblings is not unusual in our cohort. The only identified association with younger age at death in CS is early onset of cataracts $(<3$ years old $){ }^{7}$ We reevaluated this in our cohort, confirming that this association is statistically extremely significant $\left(P=1.36 \times 10^{-6}\right)$; at 5 years, survival is $\sim 60 \%$ for those patients with early cataracts and $95 \%$ for those without (Figure 4a). We also found significant associations between early cataracts and the time to development of hearing loss $\left(P=4.43 \times 10^{-7}\right)$ and contractures $(P=0.028)$, but not tremor $(P=0.86)$ or loss of subcutaneous fat $(P=0.073)$. Degree of photosensitivity (Figure $4 \mathbf{b}$ ) is not associated with survival $(P=0.23)$, or time until the onset of tremor $(P=0.398)$, though severe photosensitivity may be weakly associated with onset of hearing loss $(P=0.050)$; this feature is therefore a poor indicator of prognosis. However, severe photosensitivity does have a significant association with time until the onset of contractures $\left(P=8.31 \times 10^{-5}\right)$. We also evaluated the effect of genetic etiology on prognosis (Figure 4c). Having a CSB mutation has a borderline significant association with shorter survival $(P=0.045)$, but not with time until the onset of tremor $(P=0.078)$, hearing loss $(P=0.10)$, or contractures $(P=0.53)$. To prevent unnecessary confusion for families, we recommend that attempts at classification are avoided; instead, more descriptive information on mortality and morbidity in CS should be provided, based on the patient's clinical presentation.

Similarly, cerebro-oculofacioskeletal syndrome has been separated from CS using the following diagnostic criteria: arthrogryposis, congenital microcephaly, cataracts/microphthalmia, and severe developmental delay and growth failure. ${ }^{30}$ In essence, the main differentiating factor is the age at onset. For the purposes of management, cerebro-oculofacioskeletal may be considered part of the CS continuum. In such patients lacking CSA/CSB mutations, XPG (ERCC5) sequencing should be undertaken. ${ }^{31}$

\section{Management}

Each individual with CS should have a named, accessible clinician who is responsible for coordinating their ongoing care. Because survival beyond childhood is unusual, a pediatrician often is most appropriate. Continued involvement of a clinical geneticist is not essential but may be useful, particularly for coordinating care in adults with CS. As with other pediatric disorders, transition to adult services is problematic, not least because adults with CS are small, and many adult services are ill-equipped to manage patients of this stature. If this is the case, continued access to pediatric services should be negotiated locally if possible.

There is currently no cure for CS. The goal of surveillance in this condition is to maximize quality of life. Based on our findings, we have developed a schedule for surveillance (Table 1). Many individuals with CS remain generally well and require nonelective hospital admission only late in their disease. It is important that they become known to local clinical services prior to this, and care should be managed locally wherever possible. Clinicians seeking further information or advice on CS are welcome to contact the study team (cockayne.syndrome@ nhs.uk) and to consider recruiting their patients to this ongoing study.

\section{SUPPLEMENTARY MATERIAL}

Supplementary material is linked to the online version of the paper at http://www.nature.com/gim

\section{ACKNOWLEDGMENTS}

The authors are grateful to all participating families and to the Amy and Friends Cockayne syndrome support groups, particularly Jayne and Mark Hughes, for supporting this study. This paper is dedicated to the memory of the CS patients who passed away before publication. We are also grateful to Amy and Friends (Registered UK Charity 1119746) for their generosity in making this article available open access.

\section{DISCLOSURE}

The authors declare no conflict of interest.

\section{REFERENCES}

1. Cockayne EA. Dwarfism with retinal atrophy and deafness. Arch Dis Child 1936;11:1-8.

2. Mayne LV, Lehmann AR. Failure of RNA synthesis to recover after UV irradiation: an early defect in cells from individuals with Cockayne's syndrome and xeroderma pigmentosum. Cancer Res 1982;42:1473-1478.

3. Venema J, Mullenders LH, Natarajan AT, van Zeeland AA, Mayne LV. The genetic defect in Cockayne syndrome is associated with a defect in repair of UV-induced DNA damage in transcriptionally active DNA. Proc Natl Acad Sci USA 1990;87:4707-4711.

4. Sugita K, Suzuki N, Kojima T, et al. Cockayne syndrome with delayed recovery of RNA synthesis after ultraviolet irradiation but normal ultraviolet survival. Pediatr Res 1987;21:34-37.

5. Colella S, Nardo T, Mallery D, et al. Alterations in the CSB gene in three Italian patients with the severe form of Cockayne syndrome (CS) but without clinical photosensitivity. Hum Mol Genet 1999;8:935-941.

6. Nakazawa $Y$, Yamashita S, Lehmann AR, Ogi T. A semi-automated nonradioactive system for measuring recovery of RNA synthesis and unscheduled DNA synthesis using ethynyluracil derivatives. DNA Repair (Amst) 2010;9: 506-516.

7. Nance MA, Berry SA. Cockayne syndrome: review of 140 cases. Am J Med Genet 1992;42:68-84.

8. Natale V. A comprehensive description of the severity groups in Cockayne syndrome. Am J Med Genet A 2011;155:1081-1095.

9. Laugel V. Cockayne syndrome: the expanding clinical and mutational spectrum. Mech Ageing Dev 2013;134:161-170. 
10. Abdel Ghaffar TY, Elsobky ES, Elsayed SM. Cholestasis in patients with Cockayne syndrome and suggested modified criteria for clinical diagnosis. Orphanet J Rare Dis 2011;6:13.

11. Wilson BT, Strong A, O'Kelly S, Munkley J, Stark Z. Metronidazole toxicity in Cockayne syndrome: a case series. Pediatrics, in press.

12. Andrade LN, Nathanson JL, Yeo GW, Menck CF, Muotri AR. Evidence for premature aging due to oxidative stress in iPSCs from Cockayne syndrome. Hum Mol Genet 2012;21:3825-3834.

13. Pasquier $L$, Laugel $V$, Lazaro $L$, et al. Wide clinical variability among 13 new Cockayne syndrome cases confirmed by biochemical assays. Arch Dis Child 2006;91:178-182.

14. Ovaert C, Cano A, Chabrol B. Aortic dilatation in Cockayne syndrome. Am J Med Genet A 2007;143A:2604-2606.

15. Koob M, Laugel V, Durand $M$, et al. Neuroimaging in Cockayne syndrome. AJNR Am J Neuroradiol 2010;31:1623-1630.

16. Weidenheim KM, Dickson DW, Rapin I. Neuropathology of Cockayne syndrome: evidence for impaired development, premature aging, and neurodegeneration. Mech Ageing Dev 2009;130:619-636.

17. Neilan EG, Delgado MR, Donovan MA, et al. Response of motor complications in Cockayne syndrome to carbidopa-levodopa. Arch Neurol 2008;65: 1117-1121

18. Nardo T, Oneda R, Spivak G, et al. A UV-sensitive syndrome patient with a specific CSA mutation reveals separable roles for CSA in response to UV and oxidative DNA damage. Proc Natl Acad Sci USA 2009;106:6209-6214.

19. Horibata K, Iwamoto Y, Kuraoka I, et al. Complete absence of Cockayne syndrome group $B$ gene product gives rise to UV-sensitive syndrome but not Cockayne syndrome. Proc Natl Acad Sci USA 2004;101:15410-15415.

20. Brooks PJ. Blinded by the UV light: how the focus on transcription-coupled NER has distracted from understanding the mechanisms of Cockayne syndrome neurologic disease. DNA Repair (Amst) 2013;12:656-671.

21. Wong HK, Muftuoglu M, Beck G, Imam SZ, Bohr VA, Wilson DM 3rd. Cockayne syndrome B protein stimulates apurinic endonuclease 1 activity and protects against agents that introduce base excision repair intermediates. Nucleic Acids Res 2007;35:4103-4113.

22. Scheibye-Knudsen M, Ramamoorthy M, Sykora P, et al. Cockayne syndrome group $B$ protein prevents the accumulation of damaged mitochondria by promoting mitochondrial autophagy. J Exp Med 2012;209:855-869.

23. Aamann MD, Sorensen MM, Hvitby C, et al. Cockayne syndrome group B protein promotes mitochondrial DNA stability by supporting the DNA repair association with the mitochondrial membrane. FASEB J 2010;24:2334-2346.

24. Horibata K, Saijo M, Bay MN, et al. Mutant Cockayne syndrome group B protein inhibits repair of DNA topoisomerase I-DNA covalent complex. Genes Cells 2011;16:101-114.
25. Batenburg NL, Mitchell TR, Leach DM, Rainbow AJ, Zhu XD. Cockayne Syndrome group B protein interacts with TRF2 and regulates telomere length and stability. Nucleic Acids Res 2012;40:9661-9674.

26. Gray LT, Fong KK, Pavelitz T, Weiner AM. Tethering of the conserved piggyBac transposase fusion protein CSB-PGBD3 to chromosomal AP-1 proteins regulates expression of nearby genes in humans. PLoS Genet 2012;8:e1002972.

27. Kleijer WJ, Laugel V, Berneburg M, et al. Incidence of DNA repair deficiency disorders in western Europe: Xeroderma pigmentosum, Cockayne syndrome and trichothiodystrophy. DNA Repair (Amst) 2008;7:744-750.

28. Khayat M, Hardouf H, Zlotogora J, Shalev SA. High carriers frequency of an apparently ancient founder mutation p. Tyr322X in the ERCC 8 gene responsible for Cockayne syndrome among Christian Arabs in Northern Israel. Am J Med Genet A 2010;152A:3091-3094.

29. Falik-Zaccai TC, Laskar M, Kfir N, Nasser W, Slor H, Khayat M. Cockayne syndrome type II in a Druze isolate in Northern Israel in association with an insertion mutation in ERCC6. Am J Med Genet A 2008;146A:1423-1429.

30. Laugel V, Dalloz C, Durand M, et al. Mutation update for the CSB/ERCC6 and CSAVERCC8 genes involved in Cockayne syndrome. Hum Mutat 2010;31:113126

31. Drury S, Boustred C, Tekman M, et al. A novel homozygous ERCC 5 truncating mutation in a family with prenatal arthrogryposis-further evidence of genotypephenotype correlation. Am J Med Genet A 2014;164A:1777-1783.

32. Cole TJ, Freeman JV, Preece MA. British 1990 growth reference centiles for weight, height, body mass index and head circumference fitted by maximum penalized likelihood. Stat Med 1998;17:407-429.

This work is licensed under a Creative Commons Attribution-NonCommercial-NoDerivs $\quad 4.0$ International License. The images or other third party material in this article are included in the article's Creative Commons license, unless indicated otherwise in the credit line; if the material is not included under the Creative Commons license, users will need to obtain permission from the license holder to reproduce the material. To view a copy of this license, visit http://creativecommons.org/licenses/bync-nd/4.0/ 